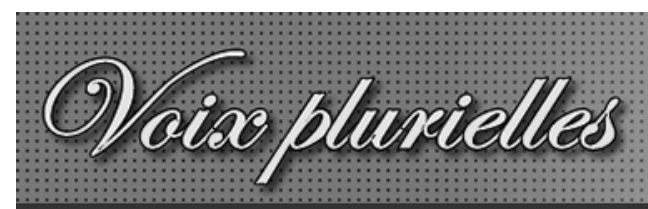

Revue de l'Association des Professeur-e-s de Français des Universités et Collèges Canadiens

\author{
Voix plurielles \\ Volume 4, Numéro 2 : septembre 2007
}

Catherine Parayre

\title{
Question d'origines ou comment préparer un corps
}

Citation MLA : Parayre, Catherine. «Question d'origines ou comment préparer un corps .» Voix plurielles 4.2 (septembre 2007).

(c) Voix plurielles, revue électronique de l'APFUCC 2007. 


\title{
Question d'origines ou comment préparer un corps
}

\author{
Catherine Parayre \\ Université Brock
}

Mai 2007

Bien que le corps et la peau soient des signes et sites identitaires, l'apparence, nous dit l'expérience, est souvent trompeuse. Les arts visuels ne manquent pas d'explorer ce paradoxe et d'en esquisser les ambiguités et l'irrésolution, chaque fois qu'ils mettent en relief les artifices de la représentation de la figure humaine, par exemple lorsque deux sources d'inspiration sont identifiables, l'une rendant présent un « réel » historique, l'autre provenant du patrimoine artistique existant. Or, l'art, « plaqué » ostensiblement sur le « réel », pour emprunter un terme employé par Henri Bergson dans un contexte différent, efface, du moins en partie, ce « réel » qui en forme la base. C'est le cas notamment chez trois artistes contemporains de réputation internationale qui pratiquent un art de l'excès ou de l'extrême appliqué à la peau et au corps, appréhendés comme le site de tous les possibles, et destiné à bouleverser les attentes et les réactions des spectateurs, à déranger leurs convictions et valeurs. Les réalisations choisies pour l'analyse sont, en cinéma, The Pillow Book (1996) de Peter Greenaway (le titre n'est pas traduit en français) ; dans les arts plastiques, les Selfhybridations précolombiennes ou africaines (1998-2002) d'Orlan; et, à la frontière de la médecine et de l'art, Le monde du corps (Körperwelten en allemand), exposition montée par l'anatomiste Gunther von Hagens, qui a débuté en 1995 à Tokyo et est, à présent, après une halte dans diverses villes européennes, en tournée en Amérique du Nord. En particulier, la citation d'œuvres d'art et la réflexion sur la collection d'art s'y conjuguent avec l'évocation de cultures exotiques, tant et si bien que le motif exotique, dont l'aspect historique est pourtant sensible dans les trois œuvres, fait place à une expression créatrice qui, nourrie de différentes traditions esthétiques et accommodée aux vues postmodernes, est caractéristique de ce qui est qualifié aujourd'hui de post-humain, de même qu'elle est située dans le cadre du musée, plutôt que dans le vécu géographique et culturel. Autrement dit, le corps exotique est, dans ces circonstances, le produit de l'invention artistique.

Certes, la notion d'exotisme soulève la question des origines, que l'origine soit un réel géographiquement distant ou une des modes européennes nées du contact avec cet ailleurs. Grevé par l'histoire du colonialisme, l'exotisme européen dans la littérature et les arts esthétise, de toute évidence, une relation inégale, dans laquelle, selon Edward Said dans son essai sur l'orientalisme, l'identité occidentale s'est constituée par contraste avec ce lointain, à partir duquel s'est formé un imaginaire et se sont définis des traits propres à l'Occident (3-5). Ces deux origines, celle géographiquement et culturellement éloignée et celle d'une pensée construite sur la notion d'un 
ailleurs, sont également discutées par des critiques qui proposent une description de l'exotisme centrée sur une approche esthétique ou sur la vision d'une condition humaine souvent utopique, telle que dans L'essai sur l'exotisme de Victor Segalen : l'exotisme « n'est autre que la notion du différent ; la perception du Divers ; la connaissance que quelque chose n'est pas soi-même ; et le pouvoir d'exotisme.... est le pouvoir de concevoir autre » (23). Se manifestant par « la perception aiguë et immédiate d'une incompréhensibilité éternelle » (25), il est « spectacle.... et... mise en beauté du spectacle » (82). Pareillement, Tzvetan Todorov conçoit l'exotisme comme une richesse trouvée chez autrui : «L'autre est à découvrir » et cette découverte ne fait pas nécessairement un objet de cet interlocuteur ; elle permet aussi de voir en lui un sujet en tous points égal (250). Et Todorov de renchérir : «Ce n'est pas faire vivre l'autre que de le laisser intact, pas plus qu'on y arrive en oblitérant entièrement sa voix » (254). En fait, dans certaines orientations littéraires et artistiques de l'avant-garde du début du vingtième siècle, en particulier le primitivisme, la thématique exotique témoignerait d'une « force d'affranchissement à l'égard de l'Occident » (Moura 161) en étant ce qui ne lui est pas soumis : «Dès le tournant du siècle, l'exotisme artistique va se déployer indéfiniment dans le vide consécutif au congé donné aux canons européens traditionnels du Beau » (389). Il est pour l'Occidental « un mouvement de retour vers une origine perdue, il métamorphose les voyages en re-découverte, et autorise la restauration d'une intimité plus pleine » (373). Qu'il soit symbole de l'hégémonie occidentale, d'une quête existentielle ou d'un travail de création, l'exotisme nous conduit vers les origines d'autrui et de nous-mêmes.

The Pillow Book, les Self-hybridations et Le monde du corps n'échappent pas à cette problématique. Bien que se déroulant en Asie, The Pillow Book, y compris sa dimension exotique, est, de l'aveu de Greenaway, un film essentiellement occidental. Faisant de fréquentes allusions à ses légendes et à son art (voir l'ouvrage de Greenaway, Pillow Book, 8-9), il montre, entre autres, différents tableaux de la Renaissance représentant saint Jérôme, non seulement car le nom est celui d'un personnage, mais aussi car saint Jérôme est connu pour son amour des livres, l'écriture étant le thème principal du film (Pascoe 166). Quant à l'élément extrême-oriental, il fait explicitement référence à des expressions artistiques européennes inspirées de l'Asie (voir Hayes sur l'influence des caractères calligraphiques dans l'art européen). L'intrigue se noue lorsque la protagoniste principale, jeune Japonaise nommée Nagiko, découvre que son père, écrivain qu'elle admire profondément et qui lui inculque l'amour de l'écriture (plusieurs passages mettent en scène le père qui, le jour de l'anniversaire de Nagiko, calligraphie des caractères sur le visage de cette dernière), accorde des faveurs sexuelles à son éditeur, afin que celui-ci le publie. Plus tard, Nagiko demandera à chacun de ses amants de répéter le geste du père (calligraphier le corps), jusqu'au jour où elle tombe amoureuse de Jérôme, traducteur britannique fasciné par l'Orient, qui l'incite à se mettre elle-même à l'écriture. Elle calligraphie alors le corps du jeune homme à plusieurs reprises. Le drame se précipite le jour où Nagiko surprend Jérôme en compagnie de l'éditeur qui, jadis, forçait son père à se prostituer. Cette nouvelle liaison cause une dispute entre les deux jeunes gens et, à sa suite, la mort de Jérôme. Désespérée et dans un dernier acte d'amour, Nagiko couvre sa dépouille de caractères calligraphiques avant que le corps soit enseveli. A son effroi, elle apprend, peu de temps après, que l'éditeur a fait exhumer le corps pour en prélever la peau, qu'il a fait ensuite préparer pour qu'elle prenne l'apparence d'un parchemin dont il ne se sépare plus. Dans l'espoir de se voir remettre les restes du jeune homme pour pouvoir lui assurer une sépulture, la 
jeune femme calligraphie la peau de plusieurs hommes, véritables livres humains, qu'elle envoie en messagers auprès de l'éditeur, mais sans succès. Elle doit attendre que l'un d'entre eux tue celuici pour qu'elle puisse enfin accomplir son dessein. Dans The Pillow Book, le corps exotique, avatar du saint Jérôme occidental, est un corps couvert de caractères de calligraphie indéchiffrables pour un Européen (ils ne sont pas toujours accompagnés de traduction), qui, de plus, évoque un récit du dixième siècle rédigé par une femme, Sei Shonagon, qui vivait à la cour, et dont certaines citations sont illustrées dans le film.

Les Self-hybridations d'Orlan révèlent l'artiste une fois transformée par des opérations chirurgicales et décorée de motifs imités de ceux figurant sur différents objets olmèques, aztèques, mayas et africains (voir Busca 24-32). Orlan écrit qu'elle s'y projette « cybernaute de ses propres visages, représentations nomades, mouvantes... sans discrimination » (Cros 166-67). Les titres sont éloquents, par exemple Sculpture Nuna, Burkina Faso, avec scarifications et corps de femme riante Euro-Stéphanoise avec bosses facio-temporales (2000), attribué à un moulage du corps de l'artiste sur lequel on été ajoutés des détails d'une statue Nuna du dix-huitième siècle appartenant au Musée du Louvre (l'adjectif « stéphanois » fait référence au lieu de naissance d'Orlan, SaintEtienne), Masque Mbangu moitié noir moitié blanc et visage de femme Euro-Stéphanoise avec bigoudis (2000) ou Défiguration-refiguration : Self-hybridation précolombienne No. 1 (1999) (voir Cros ou <http://www.orlan.net>). Dans les Self-hybridations, l'accessoire exotique, trace de l'originalité créatrice de l'artiste, met en relief les origines culturelles, euro-stéphanoises ou lointaines, non pour en fournir un commentaire, mais pour esthétiser l'apparence physique.

La question de l'exotisme se complique dans le cas du Monde du corps, qui expose des corps humains plastinés, c'est-à-dire des cadavres dans lesquels du formaldéhyde a été injecté pour empêcher toute décomposition, et qui sont ensuite préparés à des fins anatomiques (consulter $<$ http://www.bodyworlds.com>). En effet, l'identité des individus, dont le corps est arrangé dans une pose évocatrice, par exemple celle d'un joueur de basketball ou celle d'un homme portant sa peau dans la main, est jalousement gardée et la préparation des corps rend toute identification impossible. Cependant, les médias ont largement débattu de l'implantation géographique des laboratoires où sont plastinés les corps, Bichkek dans le Kirghizistan et Dalian en Chine (la plastination est interdite en Europe), et encore plus, de la provenance de certains corps, à savoir si la personne décédée avait été en mesure de donner son accord dans des pays connus pour leurs atteintes aux droits de l'individu (Posner 5). La question de l'origine et du lointain géographique est donc centrale à l'exposition, tout en étant systématiquement éludée. Plutôt que d'exotisme, on peut alors parler de post-exotisme, qui désigne un monde étranger, dans le double sens d'étrange et de lointain, en termes suffisamment abstraits pour qu'on ne puisse pas en déterminer l'origine (Volodine 11, 33 ; Huglo et Lapidus 2). Dans Le monde du corps, les corps montrés au public ont incontestablement perdu leur origine. Plus encore, avance un critique, ils sont munis d'une nouvelle identité : le joueur de basketball plastiné n'a peut-être jamais participé à ce sport, mais c'est toutefois dans ce rôle qu'il est connu du public. (Walter 4).

Par conséquent, il apparaît que la question des origines, malgré l'importance du thème du lointain, n'est pas le sujet principal de ces œuvres. En écho de la thèse de Said sur l'orientalisme, l'exotisme, qu'on en sache ou pas l'origine géographique, se situe principalement dans le geste d'appropriation de ses modèles qu'accomplit l'artiste. Sans doute la thématique exotique ou post- 
exotique n'a-t-elle ici d'autre but que de mettre en relief les stratégies artistiques et techniques de la création, la fonction première de ces œuvres consistant à faire ressortir le talent de l'artiste, qu'il s'agisse des transformations visuelles qu'Orlan imprime sur sa propre peau, des caractères dessinés sur le corps des personnages du Pillow Book afin de laisser s'épancher un sentiment d'amour et, plus tard, d'exécuter un rite funéraire, ou du savoir-faire scientifique d'un médecin et de son équipe pour conserver les corps. Par ce biais, les procédés utilisés brouillent la réflexion sur l'origine, tout en la laissant percer dans les moindres détails.

Plus pertinemment, l'intervention de l'artiste dans ces trois œuvres métamorphose le corps exotique en un corps post-humain, « reconstruit artificiellement.... pure surface où s'inscrivent les codes sociaux en cours » (Busca 44). Le résultat est un cyborg exotique, " créature faite de réalité sociale tout autant que créature de fiction » (Haraway 291, ma traduction ; sur la question du cyborg, voir van Dijck 12 pour von Hagens et Ince 90 pour Orlan). La démonstration de Donna Haraway dans son célèbre « Manifeste cyborg » s'applique aux œuvres ici étudiées de deux façons. D'une part, le mythe du cyborg illustre la « fracturation » de l'expérience (295) et « accommode » les situations et agencements les plus disparates, au point que toute interprétation d'un phénomène n'offre jamais qu'une « explication partielle » (299). D'autre part, l'écriture cyborg, corporelle, visible et lisible sur le corps, ne saurait être réduite à un code unique qu'il suffirait de décrypter, de même qu'elle n'est pas écriture des origines, préférant plutôt « déplacer » les anciens mythes et les valeurs en cours pour déstabiliser les identités (311-12).

Dans nos trois œuvres, ce principe du déplacement se décèle plus particulièrement dans la présence de nombreuses citations qui ne sauraient passer inaperçues. Rappelons que The Pillow Book transpose les symboles les plus marquants du récit de Sei Shonagon et renvoie simultanément à une riche iconographie occidentale et que l'œuvre d'Orlan est composée de références à des modèles de beauté antérieurs. Dans le cas de von Hagens, la citation concerne à la fois certains portraits d'art que la pose des plastinats copie (citons, à propos du plastinat mentionné plus haut, L'anatomie du corps humain de Juan Valverde de Amusco, gravure datant de 1559 qui représente un homme brandissant sa peau à bout de bras ; Moore et Brown 19), et diverses pratiques anatomiques depuis la dissection des cadavres par Vésale à la Renaissance jusqu'à nos jours (van Dijck 103). Ces abondantes allusions à des traditions juxtaposées pêle-mêle multiplient les codes culturels, faisant porter l'accent sur la créativité de l'artiste, et forment indéniablement un patchwork d'adaptations dont l'intérêt principal est leur agencement, plutôt que leur origine.

Une telle accumulation de citations, ajoutée à la dimension technique et/ou scientifique, puissamment stylisée, déstabilise précisément tout « effet de réel » (Barthes 174). Par exemple, Le monde du corps prête à voir une « nature fidèle à la technique » (van Dijck 21), à savoir que les procédés de plastination utilisés pour conserver les corps sont instantanément remarqués. Les corps plastinés, plutôt que d'être des représentations, sont des "imitations de représentations " (16), le modèle n'étant pas tant la « nature » ou le « réel » que d'autres corps préparés dans des salles d'anatomie et figés dans des poses symboliques ou imitant des portraits célèbres. De manière identique, le corps d'Orlan dans une pose donnée renvoie à d'autres poses de l'artiste et à son savoir-faire, tandis que les corps calligraphiés des messagers dans le film de Greenaway ont pour modèle le corps calligraphié de Jérôme mort.

Il n'est plus alors question ni d'altérité exotique ni d'humanité exotique, ou encore d'un passé 
historique, mais d'un imaginaire, presque une science-fiction postmoderne pour explorer ce qui est au-delà de la sensibilité culturelle. Ainsi Orlan a-t-elle pour projet la défiguration et la re-figuration de son propre corps :

[Elle] choisit de montrer.... un ensemble de figures empruntées aux artefacts de civilisations non occidentales : exhiber, presque objectivement, en plaquant des images exotiques sur son visage. Les portraits photographiques ou peints réalisés dans le sillage de la pénétration coloniale, visages génériques, 'types ethniques'. Afficher sans mettre en perspective.... Images de froideur esthétique, de plasticité vide, simple désir de produire un effet. (Busca 34)

L'ensemble de la carrière d'Orlan, qu'il s'agisse de la mise en scène d'opérations chirurgicales sur son propre corps afin de remodeler celui-ci en écho à des pratiques artistiques variées ou de la conception de masques, de statues et de nombreux montages photographiques dans lesquels les identités se confondent selon une technique proche du morphing, forme une réflexion approfondie sur le post-humain, que celui-ci soit exotique ou non (Busca 20-24). En fait, ce n'est pas la culture exotique qui est valorisée :

C'est elle-même qu'elle hybride, elle est champ, support et destinataire ultime. Des pièces africaines ou sud-américaines utilisées ne surnagent que quelques détails remarquables brouillés par des couleurs violentes et mis à distance par une recherche compulsive de l'étrange et de l'insolite.... Pour ce faire elle dit la surface.... [Elle] participe à la glorification postmoderne de l'artifice, de la confusion entre forme et contenu. (16)

Ce faisant, elle évite les discours idéologiques :

Elle n'affronte ni le pouvoir médical, qu'elle met en scène et glorifie, ni le marché de l'art, dont elle exploite la variété des propositions dérivées.... Elle ne s'attarde pas aux diktats imposés par la mode, l'actualité immédiate. La réflexion qu'elle mène sur les standards de beauté s'applique exclusivement à l'histoire de l'art, en Occident ou sur d'autres continents, en particulier l'Afrique et l'Amérique du Sud.... Ces lieux que l'on suppose foisonnement de conflits et de réunions se présentent quasiment désertiques. (67)

Les débats de l'exotisme demeurent également au second plan dans le Pillow Book. Au-delà du sentiment d'exotisme se manifeste dans le personnage de Nagiko la naissance d'un " sujet essentiellement postmoderne » qui survit à « sa rencontre avec la tradition littéraire judéochrétienne » qu'incarne Jérôme en sa qualité de traducteur, et révèle sa capacité à s'adapter à toutes les cultures rencontrées : en effet, Nagiko, élevée dans les traditions japonaises et chinoises, vit à Hong-Kong, présentée dans le film comme ville de toutes les avant-gardes postmodernes, de même qu'elle s'adonne à l'ancienne calligraphie orientale tout en entamant une liaison avec un Européen 
(Elliott et Purdy 277). Pour sa part, et en dépit du fait que la plastination rappelle le développement de la discipline anatomique et le spectacle de la dissection à la Renaissance, von Hagens marque la rupture avec ces pratiques ; la plastination introduit en Europe une nouvelle façon de « disposer des corps ", puisque elle n'entraîne pas sa dissolution (la momification est interdite en Europe et la dissection, par laquelle le corps est temporairement préservé, est dans tous les cas suivie de sépulture) (Walter 603-04). Le corps humain devient un site d'expérimentation scientifique sur lesquels des cyborgs futuristes se matérialisent sous la forme de corps plastinés.

Malgré l'aspect parfois sinistre d'œuvres à même la chair du sujet, l'expérience esthétique qui s'en dégage, est, doit-on toutefois convenir, somme toute rassurante. Elle est certainement celle d'un milieu protégé, le musée, non seulement dans le cas évident du Monde du corps, conçu comme une exhibition scientifique, artistique et pédagogique (606), et des Self-hybridations par une artiste dont les projets sont exposés dans le monde entier, mais également dans celui du Pillow Book, « film-musée » axé essentiellement sur le plaisir pris à agencer divers modèles artistiques et littéraires (Elliott et Purdy 268 ; voir aussi Pascoe). De fait, le musée remplit une fonction élitaire. Le plus souvent, son organisation glorifie, parfois de façon presque imperceptible, la cohésion d'une culture. Quoiqu'il soit une institution " hétérotopique », c'est-à-dire offrant la possibilité de contester des lieux réels (voir Michel Foucault, cité dans Bennett 1), le musée est formateur d'un patrimoine, comme l'atteste le développement des musées depuis le dix-huitième siècle : « S'appuyant sur une représentation magnifiée de la société et de la culture nationale et/ ou locale, [les musées] tentent d'en exprimer les caractéristiques tout autant qu'ils les inventent. En effet, en matérialisant des images mentales, ils les interprètent dans un sens positif pour la société » (Tobelem 13). En outre, le musée est un lieu dans lequel les privilèges socioculturels, en particulier celui de l'éducation, s'affirment : «Les musées trahissent, dans les moindres détails de leur morphologie et de leur organisation, leur fonction véritable, qui est de renforcer chez les uns le sentiment de l'appartenance et chez les autres le sentiment d'exclusion » (Bourdieu et Darbel 165-66). Il va sans dire que les spectateurs de nos trois œuvres découvrent l'exotisme, non dans le dépaysement géographique et culturel, mais dans l'intimité ou l'évocation d'un milieu favorisé où ils peuvent à loisir exercer le savoir culturel dont ils disposent.

Qui plus est, ces œuvres sont présentées sous forme de série, évoquant de la sorte l'agencement thématique ou autre qu'établissent les collections à l'intérieur d'un musée. Certes, elles ne constituent pas exactement des séries ou collections telles que l'entend, par exemple, Walter Benjamin, qui précise que l'objectif, quasiment encyclopédique, de la collection consiste à documenter le contexte de l'objet, qu'il s'agisse de son origine ou de son rôle (60). S'il y a série dans nos trois cas, c'est tout simplement car leurs éléments respectifs se ressemblent entre eux. Dans sa critique de la société patriarcale, les œuvres d'Orlan exploitent constamment les mêmes thèmes, parmi lesquels on peut compter les multiples transformations du corps de l'artiste, la création de palimpsestes féministes des figurations traditionnelles du corps féminin, les métaphores religieuses pour désigner l'art charnel, les titres thématiquement proches donnés aux projets, sans oublier l'effet sériel à l'intérieur de chaque projet (il existe, par exemple, une série de photographies dans laquelle elle adopte, à quelques variations près, les traits de Vénus ; une autre série dans laquelle son visage est voilé par des morceaux de gaze ou encore, bien sûr, des photographies sur lesquelles son corps arbore des motifs exotiques). Orlan déclare que, dans son travail, le mot « 'et' est un principe organisateur » 
(« Complex Dialectics », 228), c'est-à-dire que 1'art d'Orlan répète des modèles et se répète luimême. En particulier, il s'agit toujours de recomposer le corps de l'artiste sous une nouvelle facette : «Le visage d'Orlan fonctionne comme un vortex qui engloutit tout » (Busca 11). L'œuvre cinématographique de Greenaway est basée sur un semblable effet de série. "Souhaitant avant tout introduire l'esthétique de la peinture dans le cinéma et réduire l'influence de l'aspect narratif » (Gras vii, ma traduction), le réalisateur explique : « Je cherche à tout moment des alternatives à la narration. Mes films sont en grande partie basés sur des horizontales et des verticales, comme dans une grille. On y trouve des listes, des séries de nombres et des séries alphabétiques » (Petrakis 174, ma traduction). L'effet de série propre au Pillow Book est créé par l'enchaînement de gros plans, la répétition de scènes qui se font écho, l'énonciation et l'illustration de listes tirées de l'ouvrage de Sei Shonagon, le nombre d'amants que fréquente Nagiko, les tentatives d'écriture avec chacun d'eux et, à la mort de Jérôme, les corps de différents messagers envoyés à l'éditeur. Ces corps sont, d'ailleurs, dépourvus de toute individualité et ont pour seule fonction de se soumettre au désir d'écriture de la jeune femme. Finalement, l'intérêt que présente Le monde du corps réside précisément dans la seule anatomie humaine, inlassablement offerte aux regards. Dépouillés de leur identité, les corps plastinés forment une collection de corps ne différant que par la pose. L'effet de série dans ces œuvres contribue, de la sorte, à faire du corps une abstraction. Extirpé de son environnement social, le corps devient un corps pour l'art. En l'absence de concrètes relations humaines, il occupe un lieu soit symbolique soit artistique.

Or, par ce biais, il échappe à la mort. Certes, les musées commémorent souvent la disparition de pans de culture que l'on s'emploie à préserver artificiellement pour que les générations à venir n'en perdent pas le souvenir. Paul Valéry estimait que le musée abritait tout au plus des « visions mortes » (1291), alors que Theodor Adorno fait usage de l'adjectif « muséal » pour comparer le musée à un cimetière (215). Néanmoins, dans le cadre du musée, le corps mort ne cause pas l'effroi que la mort suscite usuellement. Au contraire, celle-ci apparaît souvent esthétisée ; le corps, gardé (plus ou moins) intact, immobile dans une apparence peu familière, est touché d'immortalité et l'exotisme des personnages devient ce qui dure contre nature. Dans son étude de l'exposition de von Hagens, Petra Kuppers avance que le corps, une fois préparé anatomiquement, est semblable à une carte à déchiffrer. La plastination, ajoute-t-elle, met à mort son sujet sans vraiment incarner la réalité de la mort (38). Cette analyse s'applique sans peine aux corps écrits dans The Pillow Book ou remodelés dans les Self-hybridations. D'un même point de vue, l'effet de série rend moins perceptible la souffrance que les trois œuvres laissent deviner. Orlan traite de cette question explicitement, lorsqu'elle insiste sur son refus de toute apologie de la douleur et indique ne se prêter aux opérations chirurgicales que sous l'effet d'anesthésiques, qui lui permettent toutefois de rester consciente (voir, par exemple, Weintraub 82). Sans doute la répétition inhérente à son œuvre contribue-t-elle à reléguer la question de la douleur à l'arrière-plan : on peut se dire qu'une opération insupportablement douloureuse n'aurait pas été renouvelée à plusieurs reprises. Pareillement, la violence sensible dans The Pillow Book et la douleur physique et émotionnelle qu'implique la mort dans Le monde du corps perdent leur impact dès que l'accent est mis sur le caractère sériel de chaque projet.

Exposés, sérialisés, transformés, esthétisés, déshumanisés, les corps exotiques ou postexotiques dans The Pillow Book, les Self-hybridations et Le monde du corps sont, en fin de compte, 
atemporels. D'une part, les traces du passé, du présent et du futur y sont concurremment inscrites et mêlées. D'autre part, les changements auxquels ces corps sont soumis, ne sont pas ceux du temps, mais ceux de la technique et de l'art. Ainsi, peu importe l'ancienneté des modèles choisis par Orlan ou l'âge de l'artiste ; le corps est le terrain de l'innovation et de la création. Le même bouleversement du temps caractérise l'histoire de Nagiko. En particulier, le corps de Jérôme trouble toute définition, que ce soit celle de l'exotisme - érotique car exotique européen aux yeux de Nagiko (Keesey 166), il devient à son tour exotique pour les spectateurs occidentaux une fois que Nagiko y dessine des caractères calligraphiques - ou de la temporalité - devenu parchemin, il n'est ni mort ni vivant, ni humain ni objet. De plus, le corps exotique calligraphié suggère aussi bien un ancien récit japonais que, de l'avis de Greenaway, l'avenir du cinéma, " l'innovation artistique occidentale » se mariant avec une origine orientale (149). Le film devient le musée des cultures, dans lequel l'histoire perd ses repères. Quant au monde $d u$ corps, il attire les foules et suscite souvent des réactions positives, voire enthousiastes, de la part des visiteurs, car le corps, grâce à la plastination, n'est plus condamné à la décomposition ou à l'incinération (Walter 62122). Cette nouvelle technique se révèle fascinante et rassurante, car elle promet l'immortalité du corps. Dans les trois cas, les corps exotiques sont des corps muséaux, non seulement distanciés des spectateurs parce qu'ils évoquent une culture inconnue ou méconnue, mais aussi parce que leur matérialité est inscrite dans le domaine de l'art et/ou de la science, et non dans celui du vécu. Le corps sériel exotique n'est plus tant un corps qui manifeste le lointain d'une autre culture, qu'un corps donné aux jeux de la répétition des représentations.

Sériel et exotique, le corps humain dans le travail de Greenaway, d'Orlan et de von Hagens, mesuré à l'aune de la science et des arts, placé dans des cadres culturels diversifiés, est transformé à un tel point qu'il devient un corps dont l'humanité est plus ou moins distinctement évidée. Exotique et artistique, il est un corps brouillé dont on pressent aisément que sa préparation soulève des questions éthiques, y compris portant sur la fonction et le contexte historique de l'exotisme, alors même que l'évocation au mieux ténue de la matérialité de la mort et l'esthétisation qui le caractérisent, soulignent l'abstraction et la fiction de tout objet d'art. L'humain est remplacé par l'art ; l'exotisme se réduit à un embellissement sollicitant l'attention des spectateurs et mettant en relief le nouveau et l'inconnu. Plutôt que réflexion sur l'origine, il est plus que jamais un imaginaire de tous les possibles.

\section{Ouvrages cités}

Adorno, Theodor. « Valéry Proust Museum. » Prismen. Kulturkritik und Gesellschaft. Francfort : Suhrkamp, 1976. 215-31.

Barthes, Roland. «L'effet de réel. » Essais critiques IV : Le bruissement de la langue. Paris : Seuil, 1984. 167-74.

Benjamin, Walter. « Unpacking My Library : A Talk about Book Collecting. » Illuminations : Essays and Reflections. Dir. Hannah Arendt. Trad. Harry Zohn. New York : Schocken, 1969. 59-67.

Bennett, Tony. The Birth of the Museum : History, Theory, Politics. Londres et New York : Routledge, 1995.

Bergson, Henri. Le rire : Essai sur la signification du comique. Paris : PUF, 1995. 
Bourdieu, Pierre et Alain Darbel. L'amour de l'art : Les musées d'art européens et leur public. Paris : Minuit, 1997.

Busca, Joëlle. Les visages d'Orlan : Pour une relecture du post-humain. Bruxelles : La Lettre Volée, 2002.

Cros, Caroline, Laurent Le Bon et Vivian Rehberg. « Chronophotology. » Orlan. Dir. Caroline Cros, Laurent Le Bon et Vivian Rehberg. Trad. Deke Dusinberre. Paris : Flammarion, 2004. 9-184.

Elliott, Bridget et Anthony Purdy. « Skin Deep : Fins-de-Siècle and New Beginnings in The Pillow Book. "Peter Greenaway's Postmodern/Poststructuralist Cinema. Dir. Paula WilloquetMaricondi et Mary Alemany-Galway. Lanham : The Scarecrow P, 2001. 255-81.

Gras, Marguerite. Introduction. Peter Greenaway : Interviews. Dir. Vernon Gras et Marguerite Gras. Jackson : U of Mississippi P, 2000. vii-xii.

Greenaway, Peter. The Pillow Book. Paris : Dis Voir, 1996.

Haraway, Donna. «A Cyborg Manifesto : Science, Technology, and Socialist Feminism in the Late Twentieth-Century. » The Cybercultures Reader. Dir. David Bell et Barbara M. Kennedy. London : Routledge, 2000. 291-324.

Hayes, Julie Candler. « Look but Don't Read : Chinese Characters and the Translating Drive from John Wilkins to Peter Greenaway. » Modern Language Quarterly 60.3 (1999) : 353-77.

Huglo, Marie-Pascale et Roxanne Lapidus. « The Post-Exotic Connection : Passage to Utopia. » SubStance 32.101 (2003) : 95-108.

Ince, Kate. Orlan : Millennial Female. Oxford : Berg, 2000.

Keesey, Douglas. The Films of Peter Greenaway : Sex, Death and Provocation. Jefferson, NC et Londres : McFarland, 2006.

Kuppers, Petra. The Scar of Visibility : Medical Performances and Contemporary Art. Minneapolis : U of Minnesota P, 2007.

Le monde du corps 2. Dir. Gunther von Hagens. 15 novembre 2007. <http://www.bodyworlds. com>

Moore, Charleen M. et C. MacKenzie Brown. « Gunther von Hagens and Body Worlds Part 2 : The Anatomist as Priest and Prophet. » The Anatomical Record 227B (2004) : 14-20.

Moura, Jean-Marc. La littérature des lointains : Histoire de l'exotisme européen au XXe siècle. Paris : Champion, 1998.

Orlan. Self-hybridations. 1998-2002. 15 novembre 2007. <http://www.orlan.net>

---. « The Complex Dialectics of Virtuality and Reality. » Trad. Brian Webster et Careen Irwin. Orlan. Dir. María José Kerejeta. Salamanque : Artrium, 2002. 227-29.

Pascoe, David. Peter Greenaway : Museums and Moving Images. Londres : Reaktion, 1997.

Petrakis, John. « Blasphemy in Cinema : An Interview with Peter Greenaway. » Peter Greenaway: Interviews. Dir. Vernon Gras et Marguerite Gras. Jackson : U of Mississippi P, 2000. 172-75.

Posner, Michael. «We Must Embrace Death to Understand Life. » The Globe and Mail. 20 June 2005. R5.

Said, Edward W. Orientalism. New York : Vintage, 2003.

Segalen, Victor. Essai sur l'exotisme : Une esthétique du lointain. Montpellier : Fata Morgana, 1978. 
Shonagon, Sei. The Pillow Book. Trad. Meredith McKinney. London : Penguin, 2006.

The Pillow Book. Réalisateur Peter Greenaway, avec Vivian Wu, Ewan McGregor, Ken Ogata, Yoshi Oida, Hideko Yoshida et Judy Ong. 1996. DVD. Columbia, 1997.

Tobelem, Jean-Michel. Introduction. Politique et musées. Dir. Jean-Michel Tobelem. Paris : Harmattan, 2001. 11-19.

Todorov, Tzvetan. La conquête de l'Amérique : La question de l'autre. Paris : Seuil, 1982.

Valéry, Paul. « Le problème des musées. » Euvres II. Paris : Gallimard, 1960. 1290-93.

Van Dijck, José. « Bodyworlds : The Art of Plastinated Cadavers. » Configurations 9.99-126 (2001) : 99-126.

Volodine, Antoine. Le post-exotisme en dix leçons, leçon onze. Paris : Gallimard, 1998.

Walter, Tony. « Plastination for Display : A New Way to Dispose of the Dead. » Royal Anthropology Institute 10 (2004) : 603-27.

Weintraub, Linda, Arthur Danto et Thomas McEvilley. «Self-Sanctification : Orlan. » Art on the Edge and Over : Searching for Art's Meaning in Contemporary Society 1970s-1990s. Lichtfield, CT : Arts Insights, 1996. 78-83.

Willoquet-Maricondi, Paula. "From British Cinema to Mega-Cinema. » Peter Greenaway's Postmodern/Poststructuralist Cinema. Dir. Paula Willoquet-Maricondi et Mary AlemanyGalway. Lanham : The Scarecrow P, 2001. 3-35. 